\title{
Message from the MVV Workshop Chairs
}

\author{
The Fourth International Workshop on \\ Model-Based Verification \& Validation
}

Welcome to MVV 2012 - The Fourth International Workshop on Model-Based Verification \& Validation, which is to be held in conjunction with The Sixth International Conference on Software Security and Reliability (SERE 2012) in Washington, D.C. USA from 20-22 June, 2012.

The costs entailed by software failures demonstrate that the systematic development of software at a certain level of quality is still a challenge, even after decades of research. One reason for this can certainly be found within the individual projects. Often, known techniques of quality assurance are not well understood or properly used due to deadline and budget restrictions. However, another reason is also the lack of techniques for quality assurance for specific types of software and software developed according to specific programming and modeling techniques.

Many ad-hoc security specifications, models, and protocols, which were implemented in software components in the past, turned out to be vulnerable to some extent. Formal verification and validation methods have the potential to increase user confidence in software artifacts and deliver important analytical techniques for quality assurance. In model-based verification and validation, the software under consideration is analyzed by means of a model that focuses on certain aspects, often on the behavior of the software. Models frequently used are finite state machines and flow graphs which are associated with such techniques as, for instance, formal verification, control and data flow analysis, test case generation, and model checking.

The accepted papers follow this line and present valuable verification and validation methods originating in the model-based testing area. Each paper was carefully evaluated by four reviewers. These reviews were then used for selecting papers to be presented at the conference. We would like to thank all the authors for sharing their ideas and results with us, and all the members of the Program Committee and reviewers for their help in evaluating and selecting high quality papers. Without their participation, the success of the MVV workshop would not be possible.

We thank you for attending the workshop and hope that you enjoy the program we have prepared for you.

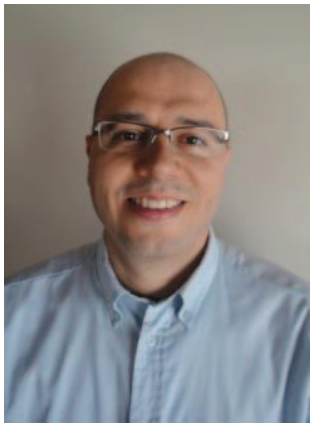

Tugkan Tuglular Izmir Institute of Technology Turkey

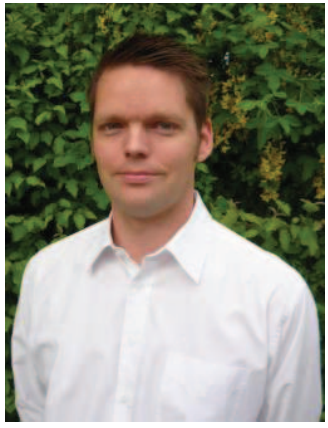

Michael Linschulte University of Paderborn Germany 